\title{
Gewichtszunahme und kolorektale Adenome
}

Hintergrund und Fragestellung: Kolorektale Adenome sind bekannte Vorläufer für die Mehrzahl der kolorektalen Karzinome (CRC). Auch eine Gewichtszunahme bei Erwachsenen wurde als Risikofaktor für CRC identifiziert. Der Zusammenhang zwischen Gewichtszunahme und der Entwicklung von kolorektalen Adenomen ist allerdings weniger gesichert. Deshalb wurde nun eine systematische Übersicht und Metaanalyse durchgeführt, um gesicherte Evidenz für diesen Zusammenhang zu gewinnen [1].

Patienten und Methodik: Mithilfe einer Medline-Suche wurden bis September 2016 prospektive Querschnitts- und retrospektive Beobachtungsstudien zur Gewichtszunahme bei Erwachsenen und das Auftreten und Wiederauftreten von kolorektalen Adenomen identifiziert. Um diese Assoziation zu spezifizieren, erfolgte eine Metaanalyse, in der eine starke Gewichtszunahme gegenüber stabilem Übergewicht sowie

Schlesinger S et al. Adult weight gain and colorectal adenomas-a systematic review and meta-analysis. Ann Oncol. 2017; 28 (6): $1217-29$
fects-Modells summiert und die 95\%-Konfidenzintervalle [95\%-KI]) berechnet.

Ergebnisse: Auf Basis von neun Studien mit 5.507 Erwachsenen betrug die summierte OR für das Auftreten von kolorektalen Adenomen 1,39 (95\%-KI 1,17 - 1,65) bei starker Gewichtszunahme (Mittel von 17,4 kg) gegenüber stabilem Übergewicht. Mit jeder Gewichtszunahme um $5 \mathrm{~kg}$ nahm das Risiko für Adenome um $7 \%$ zu ( $2-11 \%$; $=7$ Studien). Obwohl rechnerisch keine eindeutige Linearität bestand ( $p$ [für nicht linear] <0,001), war die Wahrscheinlichkeit für kolorektale Adenome durchweg über die gesamte Breite der Gewichtszunahme erhöht. In drei Studien wurde die Assoziation zwischen Gewichtszunahme und Wiederauftreten von kolorektalen Adenomen untersucht. Jedoch waren die Daten zu begrenzt, um hieraus gesicherte Schlüsse ziehen zu können.

Schlussfolgerungen: Eine Gewichtszunahme bei Erwachsenen - und sei sie auch gering - korreliert mit einem erhöhen Risiko für kolorektale Adenome. Die Daten unterstreichen die Bedeutung einer Gewichtskontrolle bei Erwachsenen im Hinblick auf das Auftreten kolorektaler Adenome. Dies ist wiederum relevant für die Prävention von CRC.

\section{- Kommentar von Ulrich R. Kleeberg, Hamburg}

\section{„Die erhebliche Bedeutung von Adipokinen und Myokinen für das Krebswachstum wird unterschätzt"}

CRC sind die dritthäufigste Krebserkrankung bei Männern und stehen an zweiter Stelle bei Frauen. Die Bedeutung verschiedener Aspekte der Lebensführung für die CRC-Inzidenz ist gesichert: Rauchen, die Ernährungsweise, körperliche Aktivität, Übergewicht und eine Gewichtszunahme im Erwachsenenalter stehen in kausalem Zusammenhang mit der Ätiologie und Pathogenese der Karzinomentwicklung (z. B. [2]). Evidenzbasiert ist auch die Adenom-zu-Karzinom-Sequenz für CRC. Daher lag es nahe, dass dieselben Risikofaktoren für die Ätiologie der Adenome ebenso relevant sind. Hinzu kommen noch der Fasergehalt der Nahrung und der Alkohol- und Fleischkonsum. Eine Metaanalyse zeigte, dass weißes, viszerales Fettgewebe eine Reihe ungünstiger metabolischer Konsequenzen hat [3], dass im viszeralen Fettgewebe gebildete Adipokine unter anderem das Wachstum kolorektaler Adenome induzieren und als Mediator für die Karzinomsequenz fungieren können. Übergewicht und Bewegungsmangel beziehungsweise die hierdurch modifizierten Adipokine des weißen Fettgewebes als wachstumsfördernde und die Myokine der Muskulatur als wachstumshemmende Zytokine haben eine erhebliche, immer noch unterschätzte Bedeutung für das Krebswachstum: Der Erfolg der Primär- und Tertiärprävention hängt entscheidend von unserer Lebensführung ab. Dies bietet eine großar- tige Möglichkeit, das eigene Schicksal günstig zu beeinflussen - die sich doch so unendlich schwer realisieren lässt. Wir dürfen nicht ermüden, diese Erkenntnisse bereits im Kindergarten zu vermitteln, und sie als Onkologen insbesondere in der Nachsorgephase von Krebspatienten ganz in das Zentrum der Bemühungen zu stellen.

\footnotetext{
Literatur

1. Schlesinger S et al. Ann Oncol. 2017; 28 (6): 1217-29

2. Huxley RR et al. Int J Cancer. 2009; 125 (1): $171-80$

3. Keum $\mathrm{N}$ et al. Ann Oncol. 2015; 26 (6): 1101 -9
}

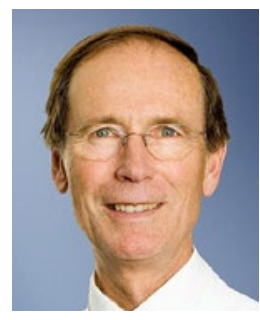

Prof. Dr. med. Ulrich R. Kleeberg

Hämatologisch-onkologische

Praxis Altona, Tagesklinik Struensee-Haus, Mörkenstr. 47, 22767 Hamburg

E-Mail: urkleeberg@hopa-hamburg.de 